九州大学学術情報リポジトリ

Kyushu University Institutional Repository

\title{
REASONING BY ANALOGY AS A PARTIAL IDENTITY BETWEEN MODELS
}

Haraguchi, Makoto

Research Institute of Fundamental Information Science, Kyushu University

Arikawa, Setsuo

Research Institute of Fundamental Information Science, Kyushu University

https://doi.org/10.5109/13381

出版情報: Bulletin of informatics and cybernetics. 22 (3/4), pp.131-147, 1987-03. Research Association of Statistical Sciences

バージョン :

権利関係 : 


\title{
REASONING BY ANALOGY AS A PARTIAL IDENTITY BETWEEN MODELS
}

\author{
By \\ Makoto Haraguchi* and Setsuo Arikawa*
}

\begin{abstract}
We present in this paper a formal theory of reasoning by analogy. We are mainly concerned with three subjects: a formal definition of analogy, a formalization of the reasoning in terms of deduction, and a method for realizing the reasoning in a logic programming system. First we assume that each domain for the reasoning is the least model for logic program. Then we consider an analogy as a partial identity between the models. Secondly we introduce a notion of rule transformation which transforms rules in one domain into those in the other. Then we can formalize the reasoning as a system with three inference rules: instantiation of rules, modus ponens, and the rule transformation. Finally, based on the formalization, we present an extended pure-Prolog interpreter which performs the detection of analogy and the reasoning by the partial identity at the same time.
\end{abstract}

\section{Introduction}

We often make use of analogy in solving various problems over various domains. Generally we have two domains. One is concerned with the current problem in inquiry, and the other is assumed to be well-known. Given such domains, we first attempt to detect some analogies between the domains, and to transform the knowledge of the well-known domain into those of the other. The transformed knowledge is not always true. However the detected analogy suggests that the knowledge may be true and that it can be used to infer some facts.

In the present paper, we are mainly concerned with the problem of formalizing the reasoning by analogy so that we can realize it by a computer. Some computational methods for the reasoning have been discussed from various viewpoints. However, since each of them has been developed for and depending on its own domain, any common framework to consider fundamental concepts about the reasoning has not been discussed. It seems to the authors that this should come from the lack of mathematical formulation of the reasoning.

From this viewpoint, one of the authors has tried to formalize the reasoning $[4,5]$. The studies are considered to be a step to reasoning by analogy by a computer. However it is true that there have still remained some problems to be overcome.

* Research Institute of Fundamental Information Science, Kyushu University 33, Fukuoka 812, Japan. 
In the previous papers $[4,5]$, the domains for the reasoning are represented by finite sets of facts (ground atoms). Given such representations, we have discussed an analogy as a partial identity between the domains. Moreover we have defined a notion of rule transformation [5] by which some ground rules in one domain are transformed to those in the other. The transformed rules are used to derive some facts. The notion of partial identity thus defined was purely syntactic, and hence it was not suited for considering the semantic aspects of analogy.

The purpose of the present paper is to modify and extend the notion of partial identity so that we can consider the semantic aspects of analogy to some extent. The new formulation is summed up as follows:

(1) The domain for the reasoning is represented by a logical formula, and is an intended model for it. In this paper, the logical formula and the intended model are assumed to be a logic program and the least (Herbrand) model for it, respectively.

(2) Given two logic programs, an analogy we consider is a partial identity between their least models. The partial identity consists of a pairing of ground terms and a one-to-one relation of facts in the domains. The paired terms are treated just like a single term in order to put two domains together.

(3) To define the reasoning based on the partial identity, we consider Winston's analogy-based reasoning $[10,11]$, in which "similar" reasons are assumed to lead to "similar" effects. We introduce a notion of rule transformation to deal with the reasoning in a framework of deduction. In fact, we can formally define the analogybased reasoning in terms of logic programming with the function of transforming rules. Then we precisely define the set of ground atoms which are reasoned based on some partial identity.

To realize the reasoning as in the above, we must design a procedure to detect a partial identity. Such a procedure plays an important role in the reasoning, since the reasoning is dependent on the chosen analogy. Here it should be noticed that the process for detecting the desired partial identity generally requires a large amount of computation. To avoid the increases of computational complexity, it is considered better to have a reasoning procedure which detects and reasons at the same time. Such a reasoning procedure has no information about the partial identity at the beginning of its computation, and it will partially get the information concerning the partial identity at each stage of reasoning process. In fact, we present a Prolog program, which is an extension of pure-Prolog interpreter, to carry out the reasoning in such a way.

In Section 2, we define the notion of partial identity and present a condition for a pairing of terms to define the partial identity. In Section 3, we formally define the reasoning based on the partial identity in terms of logic programming. In Section 4, we introduce the notion of reasoning procedure. Finally, in Section 5, we present an extended Prolog interpreter which carries out the reasoning. 


\section{Analogy as a Partial Identity}

We define in this section a notion of partial identity. Since we assume that each domain for the reasoning is represented by a logic program, we first give some necessary definitions concerning logic programs.

A definite clause is a clause of the form

$$
A \leftarrow B_{1}, \cdots, B_{n} \quad(n \geqq 0),
$$

where $A$ and $B_{j}$ are positive literals. We call the definite clause a rule. A logic program is a finite set of rules, and is simply called a program.

Since a program $P$ is a set of clauses, any model for $P$ can be considered as the corresponding Herbrand model. (For instance, see [8].) All Herbrand models for $P$ have the same domain $U(P)$, called the Herbrand universe, and the same meaning of function symbol appearing in $P . U(P)$ is defined to be the set of all ground terms whose symbols are all in $P$. Moreover the set of all terms consisting of symbols in $P$ is denoted by $L(P)$. The meaning of $n$-ary function symbol $f$ is defined to be a function $I_{f}: U(P)^{n} \rightarrow U(P)$, where

$$
I_{f}\left(t_{1}, \cdots, t_{n}\right)=f\left(t_{1}, \cdots, t_{n}\right) .
$$

We also need the notion of Herbrand base. The Herbrand base $B(P)$ of a program $P$ is defined as

$$
\begin{array}{r}
B(P)=\left\{p\left(t_{1}, \cdots, t_{n}\right) \mid p \text { is an } n\right. \text {-ary predicate symbol } \\
\text { appearing in } \left.P \text {, and } t_{j} \in U(P)\right\} .
\end{array}
$$

An element in $B(P)$ is called a ground atom.

Then, each Herbrand model (interpretation) is specified by a subset of $B(P)$. The subset consists of all ground atoms which are true under the model (interpretation). By the model intersection property [1], the intersection of all Herbrand models for $P$ is also a model for $P$. This model is called the least model for $P$, and is denoted by $M(P)$.

Proposition 2.1. ([1,8]) $M(P)$ is the set of all ground atoms which are logical consequences of $P$.

From Proposition 2.1, we take $M(P)$ as the formal meaning of $P$, and call an element in $M(P)$ a fact. In what follows, we consider only the Herbrand models, and simply call them models.

We define the correspondence of analogy by a pairing of elements in domains.

Definition 2.1. Let $P_{1}$ and $P_{2}$ be logic programs. We call a finite subset of $U\left(P_{1}\right) \times U\left(P_{2}\right)$ a pairing of terms. For a pairing $\phi$, we define the set $\psi^{+}$to be the smallest set satisfying the following conditions:

$$
\begin{aligned}
& \psi \cong \psi^{+}, \\
& \text {if }\left\langle t_{1}, t_{1}^{\prime}\right\rangle, \cdots,\left\langle t_{n}, t_{n}^{\prime}\right\rangle \in \phi^{+}, \text {then } \\
& \quad\left\langle f\left(t_{1}, \cdots, t_{n}\right), f\left(t_{1}^{\prime}, \cdots, t_{n}^{\prime}\right)\right\rangle \in \phi^{+},
\end{aligned}
$$


where $f$ is a function symbol appearing in both $P_{1}$ and $P_{2}$,

Then relation $\psi^{+}$is not always one-to-one. For example, consider the following pairing $\psi$ :

$$
\psi=\left\{\left\langle a, a^{\prime}\right\rangle,\left\langle b, b^{\prime}\right\rangle,\left\langle c, \text { on }\left(a^{\prime}, b^{\prime}\right)\right\rangle\right\},
$$

where "on" is a binary function symbols, and the other symbols appearing in $\phi$ are constant symbols. Then we have

$$
\left\langle c, \text { on }\left(a^{\prime}, b^{\prime}\right)\right\rangle,\left\langle\text { on }(a, b) \text {, on }\left(a^{\prime}, b^{\prime}\right)\right\rangle \in \phi^{+},
$$

which means that two distinct terms $o n(a, b)$ and $c$ in $P_{1}$ are related to the same term on $\left(a^{\prime}, b^{\prime}\right)$ in $P_{2}$.

As mentioned in the introduction, we consider an analogy as a partial identity. The partial identity is a function of putting together two distinct domains for the reasoning. To put them together, we first identify some paired elements, one from each domain, and treat them just like a single element. According to such an identification of elements, we identify some paired facts which hold in each domain. Thus, by the partial identity, two distinct elements in one domain are never paired with the same element in another domain. Since our domains for the reasoning are Herbrand models, distinct terms denote distinct elements. Hence the set $\psi^{+}$of paired terms should be one-to-one. The pairing $\phi$ in the example above is not allowed as a pairing of partial identity. In what follows, a pairing of partial identity is simply called a partial identity.

DEFINITION 2.2. A pairing $\phi$ is called a partial identity if $\phi^{+}$is a one-to-one relation of terms.

The paired atoms identified by the partial identity are defined as follows:

DEFINITION 2.3. For a partial identity $\phi$, two ground atoms $\alpha \in B\left(P_{1}\right)$ and $\alpha^{\prime} \in B\left(P_{2}\right)$ are said to be identified by $\psi$, denoted by $\alpha \psi \alpha^{\prime}$, if $\alpha$ and $\alpha^{\prime}$ are compatible, that is, they can be written as

$$
\begin{aligned}
& \alpha=p\left(t_{1}, \cdots, t_{n}\right), \\
& \alpha^{\prime}=p\left(t_{1}^{\prime}, \cdots, t_{n}^{\prime}\right),
\end{aligned}
$$

for some predicate symbol $p$, and $\alpha$ and $\alpha^{\prime}$ are syntactically the same up to the pairing $\phi$, that is, $\left\langle t_{i}, t_{i}^{\prime}\right\rangle \in \phi^{+}$.

Then the set of paired facts which are identified by $\phi$ is

$$
I D\left(P_{1}, P_{2} ; \psi\right)=\left\{\left\langle\alpha, \alpha^{\prime}\right\rangle \mid \alpha \in M\left(P_{1}\right), \alpha^{\prime} \in M\left(P_{2}\right), \alpha \psi \alpha^{\prime}\right\} .
$$

Proposition 2.2. ID $\left(P_{1}, P_{2} ; \phi\right)$ is a one-to-one relation of facts.

Now we present a condition for a pairing to define a partial identity. The condition is called Extended Partial Identity Condition (EPIC, for short).

Definition 2.4. A pairing $\psi$ is said to violate EPIC if there exist $\left\langle t, t^{\prime}\right\rangle,\left\langle t_{j}, t_{j}^{\prime}\right\rangle \in \psi$ and a term $T\left(X_{1}, \cdots, X_{n}\right)$ in $L\left(P_{1}\right) \cap L\left(P_{2}\right)$, that contains no constant symbols, such that

$$
t=T\left(t_{1}, \cdots, t_{n}\right) \text { and } t^{\prime} \neq T\left(t_{1}^{\prime}, \cdots, t_{n}^{\prime}\right),
$$

or

$$
t \neq T\left(t_{1}, \cdots, t_{n}\right) \text { and } t^{\prime}=T\left(t_{1}^{\prime}, \cdots, t_{n}^{\prime}\right)
$$


Moreover, if otherwise, $\phi$ is said to satisfy EPIC.

THEOREM 2.1. A pairing $\phi$ is a partial identity iff $\phi$ satisfies EPIC.

To prove the theorem, we need a definition.

DEFINITION 2.5. A pairing $\phi \subseteq U\left(P_{1}\right) \times U\left(P_{2}\right)$ is said to be a generator if there exist no $\left\langle t, t^{\prime}\right\rangle,\left\langle t_{j}, t_{j}^{\prime}\right\rangle$ in $\psi$ and a non-variable term $T\left(X_{1}, \cdots, X_{n}\right)$ containing no constant symbols such that

$$
\begin{aligned}
& t=T\left(t_{1}, \cdots, t_{n}\right), \\
& t^{\prime}=T\left(t_{1}^{\prime}, \cdots, t_{n}^{\prime}\right) .
\end{aligned}
$$

Proof of THEOREM 2.1. It is clear that $\phi^{+}$is not one-to-one if $\phi$ violates EPIC. Hence we prove only that $\phi$ violates EPIC if $\phi^{+}$is not one-to-one.

First we prove this for a generator $\phi$. Assume that $\phi$ violates EPIC, and assume that $\psi^{+}$is not one-to-one. Then there exist pairs $\left\langle t_{i}, t_{i}^{\prime}\right\rangle$ in $\psi^{+}(i=1,2)$ such that

$$
t_{1}=t_{2} \text { and } t_{1}^{\prime} \neq t_{2}^{\prime}
$$

or

$$
t_{1} \neq t_{2} \quad \text { and } \quad t_{1}^{\prime}=t_{2}^{\prime} .
$$

Assume that (2.3) holds and $\left\langle t_{j}, t_{j}^{\prime}\right\rangle \in \phi^{+} \backslash \psi(j=1,2)$. The proofs for the other cases are similar, and therefore omitted. From the assumption, there exist $\left\langle s_{j}, s_{j}^{\prime}\right\rangle,\left\langle u_{k}, u_{k}^{\prime}\right\rangle$ in $\phi$ and a non-variable term $T_{i}$ containing no constants such that

$$
\begin{array}{ll}
t_{1}=T_{1}\left(s_{1}, \cdots, s_{n}\right), & t_{1}^{\prime}=T_{1}\left(s_{1}^{\prime}, \cdots, s_{n}^{\prime}\right), \\
t_{2}=T_{2}\left(u_{1}, \cdots, u_{m}\right), & t_{2}^{\prime}=T_{2}\left(u_{1}^{\prime}, \cdots, u_{m}^{\prime}\right) .
\end{array}
$$

Case 1: $T_{1}$ and $T_{2}$ are variant. In this case, $s_{j}=u_{j}$ holds for each $j$, and there exists $i$ such that $s_{i}^{\prime} \neq u_{i}^{\prime}$. This implies that $\phi$ is not one-to-one, and therefore $\phi$ violates EPIC.

Case 2: $T_{1}$ and $T_{2}$ are not variant. Let

$$
T_{1}=T_{1}\left(X_{1}, \cdots, X_{n}\right), \quad T_{2}=T_{2}\left(Y_{1}, \cdots, Y_{m}\right) .
$$

There exists a disagreement $\left\langle V_{1}, V_{2}\right\rangle$. Here the disagreement is a symbol position at which $T_{1}$ and $T_{2}$ have distinct symbols. $V_{i}$ is the subexpression extracted from $T_{i}$ at that symbol position. Since $t_{1}=t_{2}$, one of $V_{j}$ is a variable and the other is a non-variable term. Without loss of generality, we assume that $V_{1}$ is the variable $X_{q}$. Let

$$
V_{2}=V_{2}\left(Y_{21}, \cdots, Y_{2 k}\right) \text {, }
$$

where $\left\{Y_{21}, \cdots, Y_{2 k}\right\} \subseteq\left\{Y_{1}, \cdots, Y_{m}\right\}$. Then, $t_{1}=t_{2}$ implies

$$
s_{q}=V_{2}\left(u_{21}, \cdots, u_{2 k}\right) \text {. }
$$

Since $\phi$ is a generator, this implies that

$$
s_{q}^{\prime} \neq V_{2}\left(u_{21}^{\prime}, \cdots, u_{2 k}^{\prime}\right) .
$$

Thus $\phi$ violates EPIC.

Finally, for a pairing $\psi$ which is not a generator, we consider the following generator $\psi_{0}$ : 


$$
\begin{aligned}
& \phi_{0} \leqq \psi, \\
& \phi^{+}=\phi_{0}^{+} .
\end{aligned}
$$

Let $\psi^{+}=\phi_{0}^{+}$be not one-to-one. Since $\phi_{0}$ is a generator, $\phi_{0}$ violates EPIC. From (2.5), this implies that $\phi$ violates $E P I C$, which completes the proof.

The condition EPIC concerns unifiability of terms, and plays an essential role in realizing the reasoning in a logic programming system. The details are discussed in Section 5 .

\section{Reasoning Based on Partial Identity}

In this section, we present a formal reasoning based on a partial identity. First we state the principle of reasoning by analogy:

Assume that, in $P_{1}$, the premises $\beta_{1}, \cdots, \beta_{n}$ logically imply a fact $\alpha$. Assume also that the analogous premises $\beta_{1}^{\prime}, \cdots, \beta_{n}^{\prime}$ hold in $P_{2}$. Then we reason an atom $\alpha^{\prime}$ in $P_{1}$ which is analogous to $\alpha$.

The reasoning above is conceptually due to Winston's analogy-based reasoning [11] based on the causal structures of domains. Since our analogy between $M\left(P_{1}\right)$ and $M\left(P_{2}\right)$ is a partial identity, we can restate the statement as follows:

Assume that $\beta_{1}, \cdots, \beta_{n}$ in $M\left(P_{1}\right)$ logically imply $\alpha$ in $P_{1}$, and assume that there exist $\beta_{1}^{\prime}, \cdots, \beta_{n}^{\prime}$ in $M\left(P_{2}\right)$ such that $\beta_{j} \psi \beta_{j}^{\prime}$ for all $j$. Then we reason an atom $\alpha^{\prime}$ in $B\left(P_{2}\right)$ such that $\alpha \psi \alpha^{\prime}$.

The reasoned ground atom $\alpha^{\prime}$ is not always a logical consequence of $P_{2}$. Hence the reasoning is beyond the usual deduction. Our goal is to describe the reasoning in terms of deduction. We need the following definition:

DeFinition 3.1. Let

$$
\begin{aligned}
& R_{1}=\left(\alpha \leftarrow \beta_{1}, \cdots, \beta_{n}\right), \\
& R_{2}=\left(\alpha^{\prime} \leftarrow \beta_{1}^{\prime}, \cdots, \beta_{n}^{\prime}\right)
\end{aligned}
$$

be two ground rules $(n \geqq 1)$ whose symbols are all appearing in $P_{1}$ and $P_{2}$, respectively. Let $\phi$ and $I_{j}$ be a partial identity and an Herbrand interpretation of $P_{j}$, respectively. Then the rules $R_{1}$ and $R_{2}$ are called $\psi$-analogous w. r.t. $I_{1}$ and $I_{2}$, if $\beta_{j} \in I_{1}, \beta_{j}^{\prime} \in I_{2}$, $\alpha \psi \alpha^{\prime}$, and $\beta_{j} \psi \beta_{j}^{\prime}$. In this case, $R_{1}\left(R_{2}\right)$ is called a $\psi$-analogue of $R_{2}\left(R_{1}\right)$ w. r. t. $I_{1}$ and $I_{2}$.

We call the conversion of $R_{1}$ into $R_{2}$, or $R_{2}$ into $R_{1}$, a rule transformation. In what follows, we represent the transformation by the following schema:

$$
\underset{\alpha \leftarrow \beta_{1}, \cdots, \beta_{n}}{\alpha \leftarrow \beta_{1}^{\prime}, \cdots, \beta_{n}^{\prime}}\left(\psi, I_{1}, I_{2}\right),
$$

where $\alpha \psi \alpha^{\prime}, \beta_{j} \psi \beta_{j}^{\prime}, \beta_{j} \in I_{1}, \beta_{j}^{\prime} \in I_{2}$ and the dotted line shows that the upper rule is transformed into the lower rule. By using this schema, we can represent the reasoning as follows: 


$$
\begin{gathered}
\frac{A \leftarrow B_{1}, \cdots, B_{n}}{\alpha \leftarrow \beta_{1}, \cdots, \beta_{n}}(\theta) \\
\frac{\beta_{1}^{\prime}, \cdots, \beta_{n}^{\prime} \quad}{\alpha^{\prime} \leftarrow \beta_{1}^{\prime}, \cdots, \beta_{n}^{\prime}} \\
\alpha^{\prime},
\end{gathered}
$$

where $A \leftarrow B_{1}, \cdots, B_{n}$ is a rule in $P_{1}, \theta$ is a ground substitution to obtain a logically true ground rule $\alpha \leftarrow \beta_{1}, \cdots, \beta_{n}$, and the second real line shows modus ponens. Thus the reasoning is a combination of the usual deductions and the rule transformations. This schema is said to be basic.

In general, reasoning is a process of applications of inference rules. Thus it is natural to consider a process in which the rule transformations and modus ponens are applied successively. Let us consider an example:

EXAMPLE 3.1. Let $P_{1}$ and $P_{2}$ be the following programs:

$$
\begin{aligned}
P_{1}= & \{p(a, b), \\
& q(b) \leftarrow p(a, b), \\
& r(b), \\
& s(b) \leftarrow q(b), r(b)\}, \\
P_{2}= & \left\{p\left(a^{\prime}, b^{\prime}\right),\right. \\
& \left.r\left(b^{\prime}\right)\right\} .
\end{aligned}
$$

Then we have the following basic schema:

$$
\frac{p\left(a^{\prime}, b^{\prime}\right) \frac{q(b) \leftarrow p(a, b)}{q\left(b^{\prime}\right) \leftarrow p\left(a^{\prime}, b^{\prime}\right)}}{q\left(b^{\prime}\right)}\left(\psi, M\left(P_{1}\right), M\left(P_{2}\right)\right)
$$

where $\phi=\left\{\left\langle a, a^{\prime}\right\rangle,\left\langle b, b^{\prime}\right\rangle\right\} . q\left(b^{\prime}\right)$ is not a logical consequence of $P_{2}$. However we use $q\left(b^{\prime}\right)$, as if it is a fact, to derive some additional ground atoms. In fact, we can derive $s\left(b^{\prime}\right)$ by a basic schema

$$
\frac{q\left(b^{\prime}\right), r\left(b^{\prime}\right) \frac{s(b) \leftarrow q(b), r(b)}{s\left(b^{\prime}\right) \leftarrow q\left(b^{\prime}\right), r\left(b^{\prime}\right)}}{s\left(b^{\prime}\right)}\left(\phi, M\left(P_{1}\right), M\left(P_{2}\right) \cup\left\{q\left(b^{\prime}\right)\right\}\right)
$$

Thus the successive uses of the basic schemata allow a monotonic extension of models for $P_{2}$.

Definition 3.2. For a given partial identity $\phi$, we define a set $M_{i}\left({ }^{*} ; \phi\right)$ for $i=1,2$ as follows:

$$
\begin{aligned}
& M_{i}(* ; \phi)=\bigcup_{n} M_{i}(n), \\
& M_{i}(0)=M\left(P_{i}\right)=\left\{\alpha \in B\left(P_{i}\right) \mid P_{i} \vdash \alpha\right\}, \\
& M_{i}(n+1)=\left\{\alpha \in B\left(P_{i}\right) \mid R_{i}(n) \cup M_{i}(n) \cup P_{i} \vdash \alpha\right\},
\end{aligned}
$$

where $S \vdash \gamma$ denotes that $\gamma$ is a logical consequence of $S, R_{i}(n)$ is the set of all ground rules which are $\phi$-analogues of ground instances of rules in $P_{j}(j \neq i)$ with respect to $M_{1}(n)$ and $M_{2}(n)$. 
The set $M_{i}(* ; \phi)$ is an Herbrand model for $P_{i}$. (See [7].) Hence we can assert that the reasoning based on the partial identity gives us an admissible method to extend the least model for $P_{i}$.

\section{Reasoning Procedure}

In this section, we define a reasoning procedure, and discuss some computational aspects of the reasoning based on partial identities. First we give a definition of reasoning procedure.

Definition 4.1. A reasoning procedure is an effective procedure which takes a ground atom $\alpha$ as its input and satisfies the following properties:

(1) $\alpha \in M_{i}\left(^{*} ; \phi\right)$ for some partial identity $\phi$, if it returns an answer "yes", and

(2) $\left.\alpha \oplus M_{i}{ }^{*} ; \psi\right)$ for any partial identity $\psi$, if it returns an answer " no".

DEFINITION 4.2. A reasoning procedure $M$ is complete if it returns the answer "yes" whenever $\left.\alpha \in M_{i}{ }^{*} ; \phi\right)$ for some partial identity $\phi$.

To consider a complete reasoning procedures, we present, without proof, a theorem which characterizes the reasoning defined in Section 3 in terms of deducibility. The proof is found in [7]. For a program $P_{i}$, and a partial identity $\phi$, we introduce a new predicate symbol $p_{i}$ for each predicate symbol $p$ in $P_{i}$. Moreover we define a program $\operatorname{copy}\left(P_{i}\right)$ which is obtained from $P_{i}$ by simultaneously replacing each occurrence of $p$ in $P_{i}$ by the corresponding $p_{i}$. Then we have

THEOREM 4.1. ([7]) Given $P_{i}$ and $\psi$, there exists a program $P$, denoted by $P_{1} \psi P_{2}$, such that

(1) $\operatorname{copy}\left(P_{i}\right) \subseteq P$ for $i=1,2$, and

(2) $p\left(t_{1}, \cdots, t_{n}\right) \in M_{i}(* ; \phi)$ iff $P \vdash p_{i}\left(t_{1}, \cdots, t_{n}\right)$.

Based on this theorem, we present a complete reasoning procedure $M_{G}$. Let $\phi_{1}, \phi_{2}, \cdots$ be an effective enumeration of all partial identities. Let $\langle\operatorname{dov} 1(n), \operatorname{dov} 2(n)\rangle$ be the $n$-th pair of natural numbers in an effective enumeration. Moreover, for a program $Q$ and an atom $\beta, Q \vdash{ }_{m} \beta$ denotes that $\beta$ is proved to be a logical consequence of $Q$ in at most $m$ steps of computations. Note that, in order to realize $\vdash_{m}$, it suffices to consider a complete SLD-refutation procedure $[1,8]$ with a step-counting function.

Reasoning Procedure $M_{G}$

input: ground atom $\alpha=p\left(t_{1}, \cdots, t_{n}\right)$ in $B\left(P_{1}\right) \cup B\left(P_{2}\right)$

begin

$$
\begin{array}{r}
\alpha^{\prime}:=\text { if } \alpha \in B\left(P_{1}\right) \text { then } p_{1}\left(t_{1}, \cdots, t_{n}\right) \\
\text { else } p_{2}\left(t_{1}, \cdots, t_{n}\right) ;
\end{array}
$$

$n:=1$;

while $P_{1} \phi_{\text {dov1 }(n)} P_{2} \nvdash_{d o v 2(n)} \alpha^{\prime}$ do $n=n+1$;

output the answer "yes" with $\phi_{d o v 1(n)}$

end

Then it is clear that $M_{G}$ is complete and that the set defined as 


$$
\operatorname{succ}\left(P_{1} ; P_{2}\right)=\bigcup_{n}\left\{\beta \mid P_{1} \psi_{n} P_{2} \vdash \beta\right\}
$$

is recursively enumerable.

In the next section, we present a more concrete reasoning procedure, but it is not complete.

\section{Realization of Reasoning in a Logic Programming System}

In this section, we present a reasoning procedure which is an extension of pureProlog interpreter. Given $P_{1}$ and $P_{2}$, the set $\left.M_{i}{ }^{*} ; \phi\right)$ of atoms includes the least model $M_{i}$ for $P_{i}$, and is the smallest set which is closed under applications of rules in $P_{i}$ and $R_{i}(n)$. The rules in $R_{i}(n)$ are obtained by transforming some rules in $P_{j}(j \neq i)$.

Hence it suffices to have the reasoning procedure satisfying the following properties:

(P1) It interpretes each rule in $P_{i}$ procedurally.

(P2) It performs the rule transformation based on some partial identity.

Since the domains for the reasoning are represented by logic programs $P_{i}$, a pureProlog interpreter satisfies (P1). For this reason, we design the reasoning procedure as an extension of pure-Prolog interpreter. A standard interpreter generally takes a goal of the form

$$
\leftarrow A_{1}, \cdots, A_{n},
$$

and tries to refute it by successively deriving subgoals. The rules used in the refutation are usually those in $P_{i}$. To perform the reasoning, our extended interpreter is allowed to use additional rules which should be $\phi$-analogues of rules in $P_{j}(j \neq i)$.

As mentioned in the introduction, a reasoning procedure should be designed so that it has no information about the partial identity $\psi$ at the beginning of its computation. Hence it must find a possible partial identity $\phi$ such that $\alpha \in M_{i}(* ; \phi)$.

However, given $P_{1}$ and $P_{2}$, the set (search space) of all partial identities is generally very large. Moreover, even if we introduce an ordering of partial identies, and even if we use an optimal partial identity, we still have the problem of choosing one of them.

To avoid this difficulty, we design our interpreter not to pre-compute any partial identity, but to compute it partially during the whole reasoning process. The basic idea of realizing such a reasoning process by the interpreter is briefly summed up in Section 5.1 below.

\subsection{Behavior of the extended interpreter}

Let $\alpha^{\prime}$ be a ground atom to be verified that $\alpha^{\prime} \in M_{2}(* ; \phi)$ for some partial identity $\phi$. The purpose is to find a rule in $P_{1}$, a substitution $\theta$, and a partial identity $\phi$ in the basic schema

$$
\begin{gathered}
\frac{A \leftarrow B_{1}, \cdots, B_{n}}{\alpha \leftarrow \beta_{1}, \cdots, \beta_{n}}(\theta) \\
\frac{\beta_{1}^{\prime}, \cdots, \beta_{n}^{\prime}-\frac{\beta^{\prime}-\beta_{1}^{\prime}, \cdots, \beta_{n}^{\prime}}{\alpha^{\prime}}}{\alpha^{\prime},}\left(\phi, M_{1}\left(^{*} ; \phi\right) ; M_{2}\left(^{*} ; \phi\right)\right)
\end{gathered}
$$

To obtain $\theta$, we give the interpreter a goal 


$$
\leftarrow\left[B_{1}, \cdots, B_{n} \text { in } P_{1}\right]
$$

which means the question: "Is there $\theta$ such that $B_{j} \theta \in M_{1}\left(^{*}, \phi\right)$ for some $\phi$ ?".

Suppose that our interpreter has found $\theta$. It should be noticed that, to show $\left.B_{j} \theta \in M_{1}{ }^{*}, \phi\right)$ for some $\phi$, some partial identity $\psi_{1}$ is also computed as a side effect of the interpreter. $\phi_{1}$ is empty only if $B_{j} \theta \in M\left(P_{1}\right)$. Let

$$
\begin{aligned}
& \alpha=A \theta=p\left(t_{1}, \cdots, t_{m}\right), \\
& \alpha^{\prime}=p\left(t_{1}^{\prime}, \cdots, t_{m}^{\prime}\right), \\
& B_{j} \theta=\beta_{j}=q_{j}\left(t_{j 1}, \cdots, t_{j k(j)}\right) .
\end{aligned}
$$

Since a partial identity $\psi$ has not been computed yet, we introduce variables $X_{i j}$ and consider the following transformation schema:

$$
\begin{aligned}
& p\left(t_{1}, \cdots, t_{m}\right) \leftarrow q_{1}\left(t_{11}, \cdots, t_{1 k(1)}\right), \cdots, q_{n}\left(t_{n 1}, \cdots, t_{n k(n)}\right) \\
& p\left(t_{1}^{\prime}, \cdots, t_{m}^{\prime}\right) \leftarrow q_{1}\left(X_{11}, \cdots, X_{1 k(1)}\right), \cdots, q_{n}\left(X_{n 1}, \cdots, X_{n k(n)}\right)
\end{aligned}
$$

The variable $X_{i j}$ denotes something in $U\left(P_{2}\right)$ to be paired afterward with $t_{i j} \in U\left(P_{1}\right)$. It can be instantiated by using the constraint that a set of paired term

$$
\begin{aligned}
\zeta= & \phi_{1} \cup\left\{\left\langle t_{j}, t_{j}^{\prime}\right\rangle \mid n \geqq j \geqq 1\right\} \\
& \cup\left\{\left\langle t_{i j}, X_{i j}^{\prime}\right\rangle \mid k(i) \geqq j \geqq 1, n \geqq i \geqq 1\right\}
\end{aligned}
$$

should satisfy the condition EPIC. Details of checking EPIC are discussed in Section 5.2. Let $\sigma$ be a substitution thus obtained. Then we now have a pairing $\phi_{2}=\zeta \sigma$ which is an extension and a refinement of $\phi_{1}$. From the definition of the reasoning, the next thing to do is to prove that there exists a substitution $\theta_{2}$ such that

$$
\left.q_{j}\left(X_{j 1} \sigma \theta_{2}, \cdots, X_{j k(j)} \sigma \theta_{2}\right) \in M_{2}{ }^{*}, \psi\right) .
$$

For this purpose, we generate a goal

$$
\leftarrow\left[q_{j}\left(X_{j 1} \sigma, \cdots, X_{j k(j)} \sigma\right) \text { in } P_{2}\right]
$$

and continue the whole process we have just described.

As a result, we obtain a sequence $\left\{\phi_{z}\right\}$ of sets of paired terms such that $\phi_{i+1}$ is an extension and a refinement of $\phi_{i}$. The last $\phi_{z}$ in the sequence is the desired partial identity.

\subsection{Checking the condition EPIC}

As we have discussed in Section 2, EPIC is a condition for a pairing (Definition 2.4). For the sake of simplicity, we assume here that all the function symbols but constants are unary. Then EPIC can be restated as follows:

$E P I C$ under the assumption: Given a pairing $B$, let $\left\langle t_{i}, t_{i}^{\prime}\right\rangle \in B, \operatorname{term}(X) \in L\left(P_{1}\right) \cap L\left(P_{2}\right)$. Then

(EPIC1) term $\left(t_{0}^{\prime}\right)=t_{1}^{\prime}$ whenever $\left\langle\operatorname{term}\left(t_{0}\right), t_{1}^{\prime}\right\rangle \in B$,

$(E P I C 2) \operatorname{term}\left(t_{0}\right)=t_{1}$ whenever $\left\langle t_{1}, \operatorname{term}\left(t_{0}^{\prime}\right\rangle \in B\right.$.

To check the condition (EPIC1), we consider the following nondeterministic 
procedure which computes an equation from $B$. It is completely similar to check (EPIC2), so the details of checking (EPIC2) is omitted.

Procedure $m k e q_{1}$

input: a finite set $B$ of paired terms, and a term $t$ such that $\left\langle t, t^{\prime}\right\rangle \in B$ for some $t$;

begin

choose a subterm $t_{0}$ of $t$ such that

$t=\operatorname{term}\left(t_{0}\right)$ for some $\operatorname{term}(X) \in L\left(P_{1}\right) \cap L\left(P_{2}\right)$, and $\left\langle t_{0}, t_{0}^{\prime}\right\rangle \in B$ for some $t_{0}^{\prime}$; if such a subterm is not found

then do nothing

end

else output an equation $t^{\prime}=\operatorname{ter} m\left(t_{0}^{\prime}\right)$

Let $E q_{1}(t, B)$ be the set of all equations in $U\left(P_{2}\right)$ obtained by the executions of $m k e q_{1}(t, B)$, and let

$E q_{1}(B)=\cup\left\{E q_{1}(t, B) \mid t\right.$ appears in the first argument of some pair in $\left.B\right\}$.

$E q_{2}(B)$ is similarly defined by scanning the second arguments of pairs in $B$.

EXAMPLE 5.1. Suppose that $L\left(P_{1}\right)=L\left(P_{2}\right)$, and let

$$
\begin{aligned}
B= & \{\langle f(h(a)), f(V)\rangle, \\
& \left\langle g(b), g\left(b^{\prime}\right)\right\rangle, \\
& \langle a, X\rangle, \\
& \langle h(a), Y\rangle\} .
\end{aligned}
$$

From (5.2) and (5.5), we have $f(Y)=f(V)$. From (5.2) and (5.4), we have $f(h(X))=f(V)$. Finally, from (5.4) and (5.5), we have $h(X)=Y$. As a result, we have

$$
E q_{1}(B)=\{h(X)=Y, f(h(X))=f(Y)=f(V)\} .
$$

Note that $E q_{2}(B)=\phi$ in this case.

Since we consider Herbrand interpretations, the predicate symbol "=" denotes the identity relation (on each Herbrand universe). Formally we say that $E q_{i}$ has a solution if there exists a ground substitution $\theta$ such that, for any equation $t=t^{\prime}$ in $E q_{i}, t \theta$ and $t^{\prime} \theta$ are the same term in $U\left(P_{j}\right)$, where $i \neq j$. Then we have

PROPOSITION 5.1. If there exists a substitution $\theta$ such that $B \theta \in U\left(P_{1}\right) \times U\left(P_{2}\right)$ and that $B \theta$ is a partial identity, then both $E q_{1}(B)$ and $E q_{2}(B)$ have solutions.

PROOF. Let $B$ and $\theta$ be a set of paired terms and a substitution as in the condition part of the proposition, respectively. Without loss of generality, suppose that there exist two equations in $E q_{1}(B)$ :

$$
\begin{aligned}
& t^{\prime}=\operatorname{term}_{1}\left(t_{0}^{\prime}\right), \\
& s^{\prime}=\operatorname{term}_{2}\left(s_{0}^{\prime}\right),
\end{aligned}
$$

where $\left\langle t, t^{\prime}\right\rangle,\left\langle t_{0}, t_{0}^{\prime}\right\rangle,\left\langle s, s^{\prime}\right\rangle,\left\langle s_{0}, s_{0}^{\prime}\right\rangle \in B, t=\operatorname{term}_{1}\left(t_{0}\right)$, and $s=\operatorname{term}_{2}\left(s_{0}^{\prime}\right)$. Since $B \theta$ satisfies (EPIC1), 


$$
\begin{aligned}
& t^{\prime} \theta=\operatorname{term}_{1}\left(t_{0}^{\prime} \theta\right) . \\
& s^{\prime} \theta=\operatorname{term}_{2}\left(s_{0}^{\prime} \theta\right)
\end{aligned}
$$

hold. This completes the proof.

The converse of Proposition 5.1 does not hold in general. In fact, consider a set

$$
B=\{\langle a, b\rangle,\langle g(X), Y\rangle,\langle f(g(X)), Z\rangle\},
$$

where we assume that $P_{1}$ has only $a, g$ and $f$ as its function symbols and that $P_{2}$ has only $b$ and $f$. Then we have

$$
E q_{1}(B)=\{Z=f(Y)\}
$$

This equation has a solution

$$
\theta_{n}=\left\{f^{(n+1)}(b) / Z, f^{(n)}(b) / Y\right\} .
$$

For any $\theta$ such that $B \theta \in U\left(P_{1}\right) \times U\left(P_{2}\right)$,

$$
\left\langle g(X \theta), f^{(n)}(b)\right\rangle,\left\langle f^{(n)}(a), f^{(n)}(b)\right\rangle \in(B \theta)^{+}
$$

for some $n$. Thus $B \theta$ is not partial identity.

We use Proposition 5.1 to compute a partial identity. Let $\zeta$ be the set of paired terms in (5.1), $\zeta \theta$ be a partial identity, and $E q_{1}(\zeta)=\left\{s_{i}=t_{i} \mid 1 \leqq i \leqq n\right\}$. From the proof of Proposition 5.1, the following two lists

$$
\begin{aligned}
& \text { list }_{s}=\left[s_{1}, \cdots, s_{n}\right], \\
& \text { list }_{t}=\left[t_{1}, \cdots, t_{n}\right]
\end{aligned}
$$

are unifiable by the substitution $\theta$. Let $\theta=\sigma \tau$, where $\sigma$ is the most general unifier of list $_{s}$ and list $_{t}$. Then it is clear that finding $\theta$ from $\zeta$ is reduced to finding $\tau$ from $\zeta \sigma$, which is the next set of paired terms in the sequence $\left\{\phi_{z}\right\}$.

Conversely, if list $_{s}$ and list $_{t}$ are not unifiable, then there exist no $\theta$ such that $\zeta \theta$ is a partial identity. Hence we can reject $\zeta$, and we must find another set of paired terms. The search of alternative sets of paired terms is realized in our extended interpreter by using Prolog's backtracking.

\subsection{An example of the whole reasoning process}

Now let us exemplify the whole reasoning process carried out by our extended interpreter. For this purpose, consider the following logic programs:

$$
\begin{aligned}
P_{1}= & \{f(b, c), \\
& m(a, b), \\
& g f(X, Z) \leftarrow p(X, Y), f(Y, Z), \\
& p(X, Y) \leftarrow f(X, Y), \\
& p(X, Y) \leftarrow m(X, Y)\}, \\
P_{2}= & \left\{m\left(a^{\prime}, b^{\prime}\right),\right. \\
& \left.f\left(b^{\prime}, c^{\prime}\right)\right\} .
\end{aligned}
$$


The initial goal fed to the interpreter is

$$
\leftarrow\left[g f\left(a^{\prime}, c^{\prime}\right) \text { in } P_{2}\right] \text {. }
$$

For this goal, the interpreter firstly tries to prove

$$
g f\left(a^{\prime}, c^{\prime}\right) \in M_{2}=M\left(P_{2}\right) .
$$

Clearly the interpreter fails to prove it, and therefore tries to find some $\phi$-analogue of a rule in $P_{1}$. In this case,

$$
g f(X, Z) \leftarrow p(X, Y), f(Y, Z)
$$

is chosen, since the head has the predicate symbol $g f$. The interpreter then generates a subgoal

$$
\leftarrow\left[p(X, Y), f(Y, Z) \text { in } P_{1}\right]
$$

to find the values of variables $X, Y$ and $Z$. It turns out that $X, Y$ and $Z$ are instantiated to $a, b$ and $c$, respectively, since $p(a, b), f(b, c) \in M_{1}$. Hence, at this point, the flrst set $\phi_{1}$ of paired terms is empty. Based on the substitution above, the interpreter produces a rule transformation.

$$
\frac{g f(a, c) \leftarrow p(a, b), f(b, c)}{g f\left(a^{\prime}, c^{\prime}\right) \leftarrow p\left(X_{1}, X_{2}\right), f\left(X_{3}, X_{4}\right)}
$$

where the variables $X_{j}$ are introduced to compute a partial identity. Then we have a set

$$
B_{1}=\left\{\left\langle a, a^{\prime}\right\rangle,\left\langle c, c^{\prime}\right\rangle,\left\langle a, X_{1}\right\rangle,\left\langle b, X_{2}\right\rangle,\left\langle b, X_{3}\right\rangle,\left\langle c, X_{4}\right\rangle\right\},
$$

and ask then the existence of a substitution $\theta$ such that $B_{1} \theta$ is a partial identity. Our extended interpreter computes

$$
\begin{aligned}
& E q_{1}=\left\{a^{\prime}=X_{1}, X_{2}=X_{3}, c^{\prime}=X_{4}\right\}, \\
& E q_{2}=\phi
\end{aligned}
$$

from $B_{1}$. From these equations, we have two lists

$$
\left[a^{\prime}, X_{2}, c^{\prime}\right], \quad\left[X_{1}, X_{3}, X_{4}\right] .
$$

Unifying them, we have the most general unifier

$$
\theta=\left\{a^{\prime} / X_{1}, X_{3} / X_{2}, c^{\prime} / X_{4}\right\} .
$$

Hence the next set $\phi_{2}$ of paired terms is

$$
\phi_{2}=\left\{\left\langle a, a^{\prime}\right\rangle,\left\langle b, X_{3}\right\rangle,\left\langle c, c^{\prime}\right\rangle\right\} .
$$

We now have a rule transformation

$$
\frac{g f(a, c) \leftarrow p(a, b), f(b, c)}{g f\left(a^{\prime}, c^{\prime}\right) \leftarrow p\left(a^{\prime}, X_{3}\right), f\left(X_{3}, c^{\prime}\right)}
$$

The value of $X_{3}$ is still unknown, so the interpreter generates the next subgoal 


$$
\leftarrow\left[p\left(a^{\prime}, X_{3}\right), f\left(X_{3}, c^{\prime}\right) \text { in } P_{2}\right]
$$

to find the value. Since there exist no instance of $X_{3}$ such that $p\left(a^{\prime}, X_{3}\right) \in M_{2}$, the interpreter similarly tries to find $\phi$-analogue of a rule in $P_{1}$. In this case, there exist two rules

$$
\begin{aligned}
& \text { (R1): } p(X, Y) \leftarrow f(X, Y), \\
& \text { (R2): } p(X, Y) \leftarrow m(X, Y)
\end{aligned}
$$

which have the predicate symbol $p$ in their head parts. These two possibilities are examined alternatively. First suppose that (R1) is chosen. Our interpreter instantiates $X$ and $Y$, and a rule transformation:

$$
\begin{gathered}
p(b, c) \leftarrow f(b, c) \\
p\left(a^{\prime}, X_{3}\right) \leftarrow f\left(X_{4}, X_{5}\right)
\end{gathered}
$$

where $X_{4}$ and $X_{5}$ are new variables. From the schema above, we have

$$
\sigma_{1}=\left\{\left\langle b, a^{\prime}\right\rangle,\left\langle c, X_{3}\right\rangle,\left\langle b, X_{4}\right\rangle,\left\langle c, X_{5}\right\rangle\right\},
$$

and the next set of paired terms

$$
\sigma_{1} \cup \phi_{2}=\left\{\left\langle a, a^{\prime}\right\rangle,\left\langle b, X_{3}\right\rangle,\left\langle c, c^{\prime}\right\rangle,\left\langle b, a^{\prime}\right\rangle,\left\langle c, X_{3}\right\rangle,\left\langle b, X_{4}\right\rangle,\left\langle c, X_{5}\right\rangle\right\} .
$$

In this case,

$$
a=b \in E q_{2}\left(\sigma_{1} \cup \psi_{2}\right),
$$

which is not unifiable. Hence our extended interpreter goes back to (5.8). Now it chooses (R2) instead of (R1).

Similarly we have a rule transformation:

$$
\frac{p(a, b) \leftarrow m(a, b)}{p\left(a^{\prime}, X_{3}\right) \leftarrow m\left(X_{4}, X_{\overline{0}}\right)}
$$

with the new set of paired terms

$$
\sigma_{2}=\left\{\left\langle a, a^{\prime}\right\rangle,\left\langle b, X_{3}\right\rangle,\left\langle a, X_{4}\right\rangle,\left\langle b, X_{5}\right\rangle\right\} .
$$

Then we have

and

$$
\phi_{2} \cup \sigma_{2}=\left\{\left\langle a, a^{\prime}\right\rangle,\left\langle b, X_{3}\right\rangle,\left\langle c, c^{\prime}\right\rangle,\left\langle a, X_{4}\right\rangle,\left\langle b, X_{5}\right\rangle\right\},
$$

$$
E q_{1}\left(\phi_{2} \cup \sigma_{2}\right)=\left\{a^{\prime}=X_{4}, X_{3}=X_{5}\right\} .
$$

Unifying $\left[a^{\prime}, X_{3}\right]$ and $\left[X_{4}, X_{5}\right]$, we have a rule transformation

$$
\begin{gathered}
p(a, b) \leftarrow m(a, b) \\
p\left(a^{\prime}, X_{5}\right) \leftarrow m\left(a^{\prime}, X_{5}\right)
\end{gathered}
$$

and

$$
\phi_{3}=\left\{\left\langle a, a^{\prime}\right\rangle,\left\langle b, X_{5}\right\rangle,\left\langle c, c^{\prime}\right\rangle\right\} .
$$

To find the value of $X_{5}$, the goal

$$
\leftarrow\left[m\left(a^{\prime}, X_{5}\right) \text { in } P_{2}\right]
$$


is generated, and it succeeds with $b^{\prime} / X_{5}$, since $m\left(a^{\prime}, b^{\prime}\right) \in M_{2}$. Thus we have a partial identity

$$
\phi=\left\{\left\langle a, a^{\prime}\right\rangle,\left\langle b, b^{\prime}\right\rangle,\left\langle c, c^{\prime}\right\rangle\right\}
$$

and complete to prove $g f\left(a^{\prime}, c^{\prime}\right) \in M_{\mathbf{2}}(* ; \phi)$.

\subsection{Control structure of the extended interpreter}

We now present our interpreter called "ana" in a form of Prolog program. First suppose that a fact of assertion $\mathrm{A}$ and a rule $C \leftarrow B_{1}, \cdots, B_{n}$ in $P_{1}$ are represented by Prolog's clauses

$$
\begin{aligned}
& \operatorname{fact}_{1}(A) . \\
& \operatorname{fact}_{1}(C):-\operatorname{fact}_{1}\left(B_{1}\right), \cdots, \operatorname{fact}_{1}\left(B_{n}\right) .,
\end{aligned}
$$

respectively, and stored in Prolog database, where fact $_{1}$ is a reserved symbol to show that the fact and the rule are in $P_{1}$. For a clause in $P_{2}$, we assume a similar representation using a symbol fact $_{2}$. Now we list the Prolog program ana.

(C1) ana (Goal):-reason (Goal, [ ], Epic).

(C2) reason (true, Epic, Epic):- !.

(C3) reason ((Goal, Goals), Epic, Epic2)

:- !, reason (Goal, Epic, Epic1), reason (Goals, Epic1, Epic2).

(C4) reason (Goal, Epic, Epic1)

$$
\begin{aligned}
\text { :- } & \text { clause (Goal, Goals), } \\
& \text { reason(Goals, Epic, Epic1). }
\end{aligned}
$$

(C5) reason (Goal, Epic, Epic3)

$$
\begin{aligned}
\text { :- } & \text { prematch (Goal, Sgoal, Sgoals), } \\
& \text { reason (Sgoal, Sgoals, Tgoals, Bind), } \\
& \text { trans (Goal, Sgoal, Sgoals, Tgoals, Bind), } \\
& \text { epic (Bind), } \\
& \text { append }(E p i c 1, \text { Bind, Epic } 2), \\
& \text { epic }(E p i c 2), \\
& \text { reason }(T g o a l s, \text { Epic2, Epic } 3), \\
& \text { epic }(E p i c 3) .
\end{aligned}
$$

A goal $\leftarrow\left[A\right.$ in $\left.P_{1}\right]$ fed to our interpreter is represented by a Prolog's term $\operatorname{fact}_{i}(A)$. Hence the initial goal to the Prolog interpreter is

$$
\leftarrow a n a\left(\operatorname{fact}_{i}(A)\right) \text {. }
$$

Some set of paired terms are to be assigned to the second and the third arguments of the predicate reason. A set of paired terms $\left\{\left\langle t_{j}, t_{j}^{\prime}\right\rangle \mid 1 \leqq j \leqq n\right\}$ is represented by a pair-list $\left[\left[t_{1}, t_{1}^{\prime}\right], \cdots,\left[t_{n}, t_{n}^{\prime}\right]\right]$. The empty list is denoted by [] . Given a goal and a pair-list list $_{0}$ assigned to the variable Epic, reason computes a pair-list list $_{1}$ which is an extension and a refinement of list $_{0}$. The third variable Epic ${ }^{\prime}$ of reason is instantiated to list $_{1}$, when the goal reason (Goal, Epic, Epic') succeeds. The process of finding the sequence of $\left\{\psi_{k}\right\}$ is thus realized by a recursion. 
The ordered set of clauses (C2), (C3) and (C4) works as a pure-Prolog interpreter. The last clause (C5) carries out the rule transformation and the construction of pairlist. The predicate prematch in the body of (C5) searches a possible rule in $P_{j}(j \neq i)$ to be transformed.

The predicate trans produces a pair-list necessary to perform transformations. The produced pairing is assigned to the variable Bind.

The predicate epic computes a set of equations, and tries to unify them, as we have discussed. Hence, after the execution of epic(Bind), some variables in Bind are instantiated to terms as long as Bind does not violate the condition EPIC. If it turns out that Bind violates $E P I C$, reason goes back, and tries to find another pair-list and another rule to be transformed.

Finally we present an example (due to Winston [11]) of the reasoning performed by the Program "ana". In the example, the question asked to ana is

$$
\leftarrow\left[\operatorname{kill}(X, \text { noble }-a) \text { in } P_{2}\right] \text {. }
$$

Equivalently it has the form

$$
\operatorname{ana}\left(\operatorname{fact}_{2}\left(k i l l\left(X, \text { noble }_{-} a\right)\right)\right)
$$

as an initial goal to the Prolog interpreter. The underlying logic programs for the reasoning are as follows:

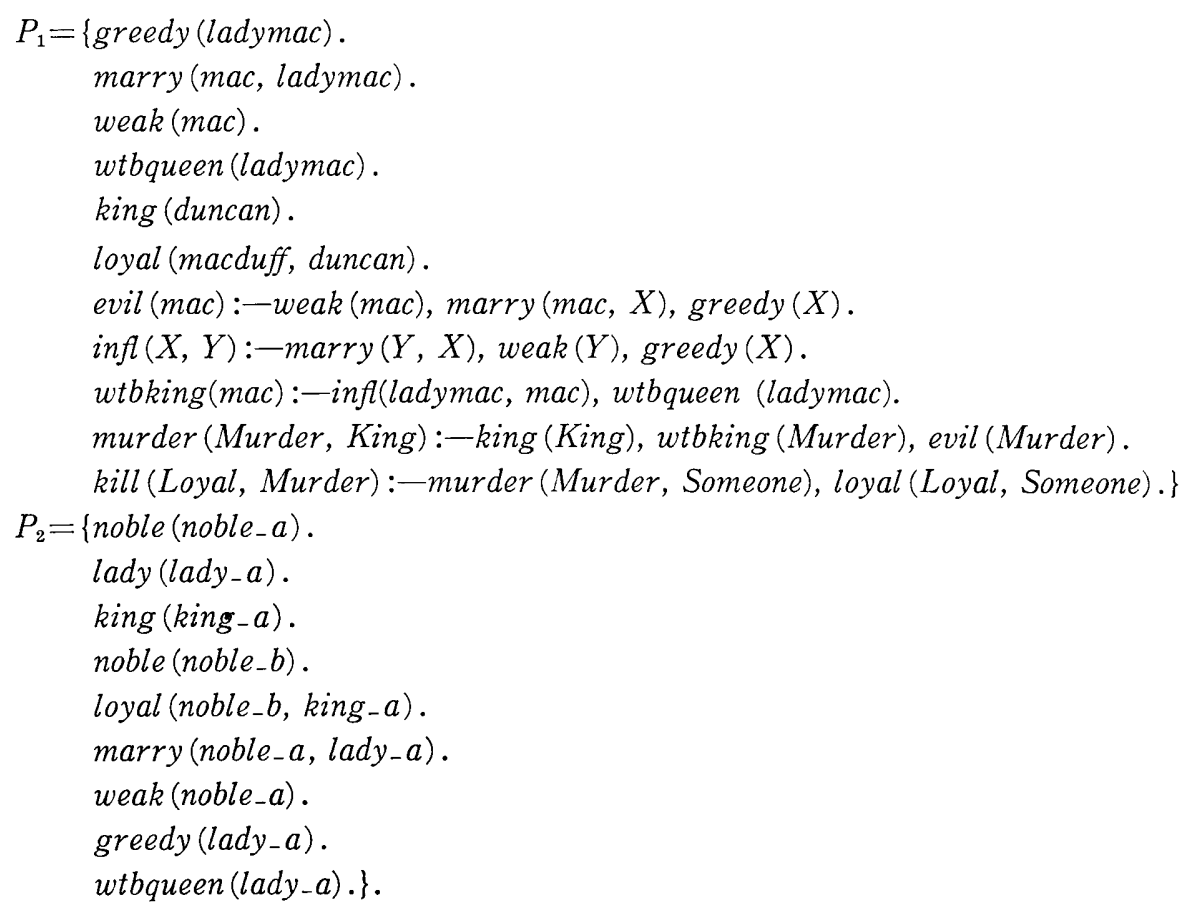

The extended interpreter has been developed for DCL U-station in CProlog, and returned the answer

$$
X=\text { noble }_{-} b
$$


for the question above after about 15 seconds.

\section{References}

[1] APt, K.R. and VAn EmDen, M.H.: Contribution to the theory of logic programming, JACM, 29, 3 (1982), 841-862.

[2] Clark, K. L.: Negation as Failure, Logic and Databases, H. Gallaire and J. Minker (Eds.), Plenum Press, New York (1978), 293-322.

[3] Gentner, P.: Are scientific analogies metaphors?, Metaphor: Problems and Perspectives, D. S. Miall (Ed.), The Harvester Press, Sussex (1982), 106-132.

[4] Haraguchi, M.: Towards a mathematical theory of analogy, Bull. Inform. Cybernetics, 21 (1985), 29-56.

[5] HARAGUCHI, M.: Analogical reasoning using transformations of rules, LNCS 221 (1986), $56-65$.

[6] Haraguchi, M. and Arikawa, S.: A formulation and a realization of analogical reasoning, Journal of JSAI, 1, 1 (1986), 132-139 (in Japanese).

[7] Haraguchi, M. and Arikawa, S.: Analogical Union of Logic Programs, Proc. Logic Programming Conference '86 (1986), 103-110.

[8] LLOYD, J.W.: Foundations of logic programming, Springer-Verlag (1984).

[9] POLYA, G.: Induction and analogy in mathematics, Princeton University Press (1954).

[10] Winston, P.H. : Learning and reasoning by analogy, CACM, 23 (1980), 689-703.

[11] Winston, P. H.: Learning new principles from precedents and exercises, Artificial Intelligence, 19 (1983), 321-350.

Received September 29, 1986 